\title{
Orally administered $L$. lactis secreting an anti-TNF Nanobody demonstrate efficacy in chronic colitis
}

\author{
K Vandenbroucke $^{1,2,3}, \mathrm{H}$ de Haard ${ }^{4}$, E Beirnaert ${ }^{4}$, T Dreier ${ }^{4}$, M Lauwereys $^{4}$, L Huyck $^{1,2}$, J Van Huysse ${ }^{5}$, \\ P Demetter $^{6}$, L Steidler $^{3}$, E Remaut $^{1,2}$, C Cuvelier $^{5}$ and P Rottiers ${ }^{1,2,3}$
}

Inflammatory bowel disease (IBD) is a chronic inflammatory gastrointestinal disorder. Systemic treatment of IBD patients with anti-tumor necrosis factor (TNF)- $\alpha$ antibodies has proven to be a highly promising approach, but several drawbacks remain, including side effects related to systemic administration and high cost of treatment. Lactococcus lactis was engineered to secrete monovalent and bivalent murine (m)TNF-neutralizing Nanobodies as therapeutic proteins. These therapeutic proteins are derived from fragments of heavy-chain camelid antibodies and are more stable than conventional antibodies. L. lactis-secreted anti-mTNF Nanobodies neutralized mTNF in vitro. Daily oral administration of Nanobody-secreting $L$. lactis resulted in local delivery of anti-mTNF Nanobodies at the colon and significantly reduced inflammation in mice with dextran sulfate sodium (DSS)-induced chronic colitis. In addition, this approach was also successful in improving established enterocolitis in interleukin 10 (IL10 $)^{-/-}$mice. Finally, L. lactis-secreted anti-mTNF Nanobodies did not interfere with systemic Salmonella infection in colitic IL10 ${ }^{-/-}$mice. In conclusion, this report details a new therapeutic approach for treatment of chronic colitis, involving in situ secretion of anti-mTNF Nanobodies by orally administered $L$. lactis bacteria. Therapeutic application of these engineered bacteria could eventually lead to more effective and safer management of IBD in humans.

\section{INTRODUCTION}

Inflammatory bowel disease (IBD) is a family of chronic disorders that cause inflammation of the digestive tract. ${ }^{1}$ Tumor necrosis factor- $\alpha$ (TNF) is believed to be responsible for many of the clinical symptoms in IBD. ${ }^{2,3}$ With a prevalence of $2 / 1,000$, IBD is becoming a significant problem in the Western world.

Systemic treatment of IBD patients with anti-TNF antibodies has become an established therapy for Crohn's disease and ulcerative colitis. Intravenous infusion of Infliximab (Remicade, Centocor Inc., Malvern, PA), a monoclonal chimeric anti-TNF antibody, was the first and currently best studied Food and Drug Administration-approved anti-TNF therapy for Crohn's disease and ulcerative colitis. ${ }^{4-7}$ Although repeated intravenous administration of Infliximab can be very efficacious, ${ }^{5,6}$ treatment is costly, has poor compliance, and can be associated with serious adverse events. ${ }^{8-16}$ As many of these unwanted effects are linked to systemic application, they might be resolved by local delivery at the site of inflammation.
Previously, we described a system (TopAct) for the oral delivery of biopharmaceuticals to the intestine, based on local synthesis and delivery of therapeutic proteins by viable genetically modified Lactococcus lactis strains (ActoBiotics) in the gut, in preclinical experiments as well as a clinical trial. ${ }^{17-21}$

Coppieters et al. ${ }^{22}$ developed formatted anti-TNF singledomain antibody fragments (Nanobodies), ${ }^{23,24}$ derived from heavy-chain camelid antibodies, as therapeutic agent in murine rheumatoid arthritis. These molecules can be cloned and produced easily as recombinant proteins in bacteria and yeast ${ }^{24,25}$ and are able to overcome various issues often encountered with classical antibodies. ${ }^{26,27}$ Furthermore, Nanobodies constitute modular building blocks suitable to generate bispecific and bivalent antibody constructs. ${ }^{26}$

\section{RESULTS}

In vitro synthesis of MT1 and MT1-MT1 by L. lactis

Synthesis of MT1 and MT1-MT1 Nanobodies by L. lactis was evaluated by Western blot (Figure 1a) and enzyme-linked 
a

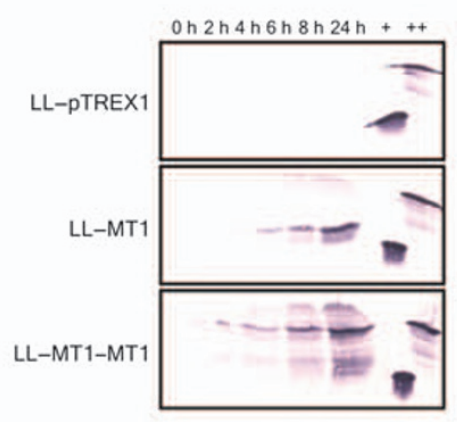

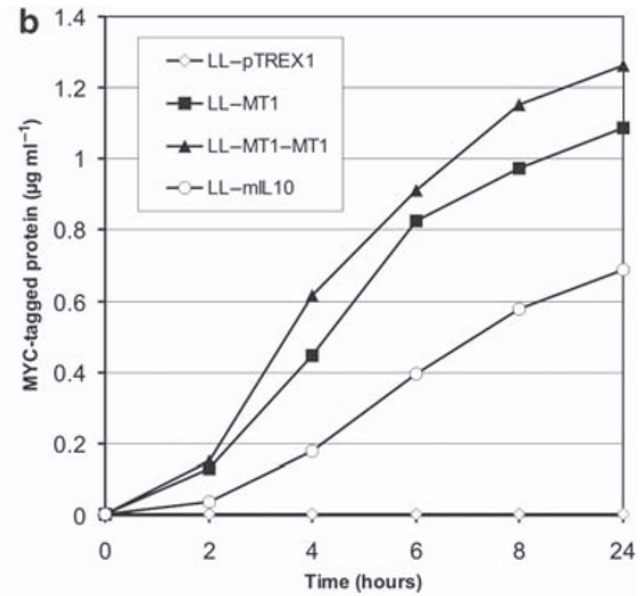
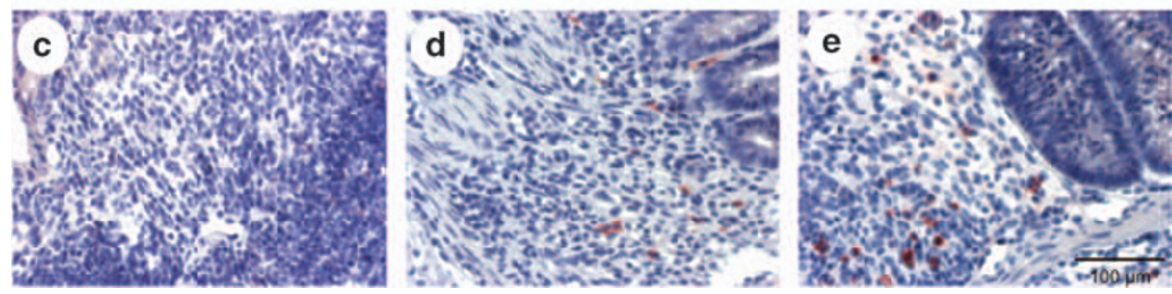

Figure 1 In vitro and in vivo production of monovalent and bivalent MT1 and MT1-MT1 Nanobodies by L. lactis (LL-MT1 and LL-MT1-MT1, respectively). (a) Western blot analysis of proteins secreted in vitro by the various strains, revealed by anti-Nanobody rabbit antibody K208. Each lane on the blot represents $250 \mu \mathrm{l}$ of $\mathrm{L}$. lactis culture supernatant obtained after different periods of growth $\left(2 \times 10^{7} \mathrm{CFU}\right.$ at time zero). A $250 \mathrm{ng}$ purified monovalent MT1 $(+)$ and bivalent MT1-MT1 $(++)$ were used as positive control. (b) Concentrations of secreted heterologous Myc-tagged proteins

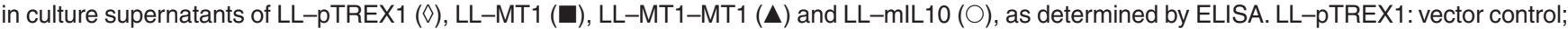
LL-mIL10: L. lactis strain secreting mIL10. (c-e) Representative immunohistochemical images for the detection of anti-TNF Nanobodies in the distal colon of mice with DSS-induced colitis, treated with empty expression vector LL-pTREX1 (c), LL-MT1 (d) or LL-MT1-MT1 (e). CFU, colony-forming units; DSS, dextran sulfate sodium; ELISA, enzyme-linked immunosorbent assay; mIL10, murine interleukin 10. L. lactis; Lactococcus lactis.

immunosorbent assay (ELISA, Figure 1b). After $24 \mathrm{~h}$ of growth, MT1 and MT1-MT1 were found in the culture supernatant at concentrations of $1080 \pm 135 \mathrm{ng} \mathrm{ml}^{-1}$ and $1264 \pm 144 \mathrm{ng} \mathrm{ml}^{-1}$, respectively. Constitutive Nanobody secretion did not alter L. lactis growth rate.

\section{In vivo synthesis of MT1 and MT1-MT1 by L. lactis}

Ten serial inoculations, containing vehicle or $2 \times 10^{9} \mathrm{CFU}$ LL-pTREX1, LL-MT1, or LL-MT1-MT1, were administered to mice with dextran sulfate sodium (DSS)-induced colitis at intervals of $30 \mathrm{~min}$. One hour after the last inoculation, mice were analyzed. Colony-forming units (CFU) values of the various L. lactis strains in the entire colon averaged $5 \times 10^{8}$ (Table 1). The Nanobody quantification protocol detected $6.5 \pm 0.7 \mathrm{ng} \mathrm{MT1}$ and $9.7 \pm 1.4 \mathrm{ng}$ MT1-MT1 per colon (Table 1) whereas no Nanobodies could be retrieved from systemic circulation. Furthermore, immunohistochemical detection of Nanobodies in the distal part of the colon of DSS-induced colitic mice clearly showed that anti-TNF Nanobodies were present in the mucosa of mice treated with LLMT1 and LL-MT1-MT1. The Nanobodies were also observed to be associated with the surface of lamina propria cells, especially in eroded zones with a dominant inflammatory infiltrate (Figure 1d and e). Nanobodies could not be detected in controls (Figure 1c). This indicated that L. lactis strains LL-MT1 and LL-MT1-MT1 actively produced MT1 and MT1-MT1, respectively, in vivo and delivered them efficiently to the colonic mucosa.

\section{L. lactis-secreted Nanobody MT1-MT1 is bioactive and inhibits the actions of both soluble TNF and transmembrane TNF}

Both E. coli-produced, purified Nanobodies, as well as MT1 and MT1-MT1 Nanobodies secreted by L. lactis were able to neutralize soluble TNF (Figure 2a).

The ability of MT1 and MT1-MT1 to counteract the cytotoxic effects of transmembrane (tm)TNF was subsequently investigated. The effect of MT1 was less pronounced, both with the E. coli produced, purified, and the L. lactis-secreted form, but the effect of bivalent Nanobody MT1-MT1 was clear in both cases (Figure 2b). Given the fact that bivalent MT1-MT1 neutralized tmTNF and had a higher soluble TNF neutralization capacity than monovalent MT1, the proceeding experiments focused on bivalent MT1-MT1 Nanobodies.

\section{Therapeutic effect of LL-MT1-MT1 against DSS-induced chronic colitis}

Healthy mice showed an average histological score of $0.9 \pm 0.2$ in the middle colon (Figure 3a and f). Vehicle-treated control mice and the LL-pTREX1 control group showed a mean histological score of $5.5 \pm 0.4$ and $5.4 \pm 0.3$, in the middle colon, respectively (Figure $3 \mathbf{b , c}$ and f). The mean histological score of LL-MT1-MT1-treated mice $(3.6 \pm 0.3 ; P<0.01$; Figure $3 \mathbf{d}$ and $\mathbf{f})$ was at least $30 \%$ lower than that of vehicle-treated and empty vector-treated control groups. The efficacy of LL-MT1-MT1 treatment against chronic DSS-induced colitis was comparable with that observed in LL-mIL10-treated 
Table 1 In vivo detection of administered $L$. lactis bacteria and their secreted products

\begin{tabular}{lcccc}
\hline & PBS & LL-pTREX1 & LL-MT1 & LL-MT1-MT1 \\
\hline CFU & $0.20 \times 10^{1}$ & $5.24 \times 10^{8}$ & $5.72 \times 10^{8}$ & $4.04 \times 10^{8}$ \\
MT1 or MT1-MT1 & $0.00 \pm 0.00$ & $0.0 \pm 0.02$ & $6.54 \pm 0.68$ & $9.66 \pm 1.38$ \\
(ng per colon) $^{a}$ & & & & \\
\hline
\end{tabular}

${ }^{a}$ Amount of MT1 or MT1-MT1 Nanobodies in ng per colon \pm s.d. CFU, colony forming units; L. lactis, Lactococcus lactis; PBS, phosphate-buffered saline.
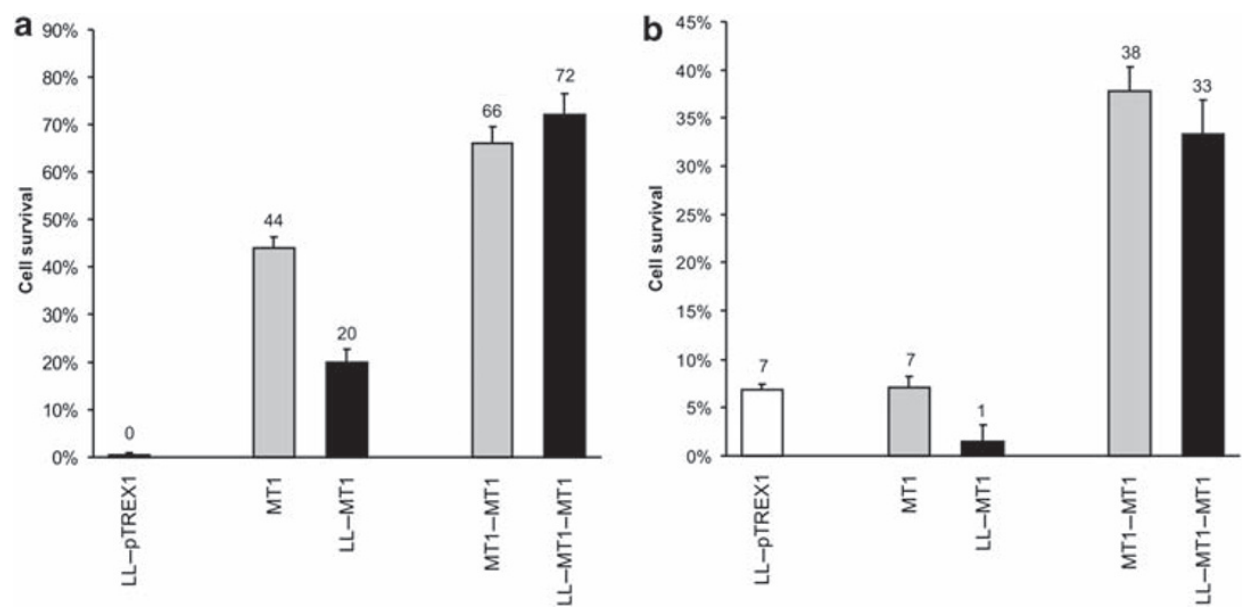

Figure 2 Effect of E. coli-produced, purified and L. lactis-secreted monovalent and bivalent MT1 and MT1-MT1 Nanobodies on the cytotoxic actions of soluble TNF and tmTNF. (a) Soluble TNF $\left(20 \mathrm{Uml}^{-1}\right)$ was neutralized by MT1 and MT1-MT1 in an $18 \mathrm{~h}$ cytotoxicity assay using WEHI 164 clone 13-cells in the presence of $1 \mu \mathrm{g} \mathrm{ml}^{-1}$ actinomycin D. (b) E. coli-produced, purified and L. lactis-secreted Nanobody MT1-MT1 was able to inhibit the cytotoxic effects of L929 cells that express uncleavable tmTNF on WEHI 164 clone 13-cells in the presence of $1 \mu \mathrm{g} \mathrm{ml}^{-1}$ actinomycin D. The gray bars represent wells in which purified MT1 or MT1-MT1 was added (total concentration of $250 \mathrm{ng} \mathrm{ml}^{-1}$ ). The black bars represent wells in which $50 \mu$ l of filtered $(0.22 \mu \mathrm{m})$ lactococcal supernatant was added. The final concentration of $L$. lactis-secreted MT1 or MT1-MT1 was $250 \mathrm{ng} \mathrm{ml}{ }^{-1}$ in each setting. E.coli, escherichia coli; L. lactis, Lactococcus lactis; tm, transmembrane; TNF, tumor necrosis factor.

mice, where a mean histological score of $3.5 \pm 0.4$ was noted $(P<0.01$ compared with mock-treated and empty vector-treated control groups; Figure $3 \mathbf{e}$ and f). In conclusion, LL-MT1-MT1 treatment efficiently ameliorated DSS-induced chronic colitis in mice.

\section{Therapeutic effect of LL-MT1-MT1 against established chronic enterocolitis in interleukin $10^{-1-}$ mice}

Treatment with LL-MT1-MT1 caused a significant decrease in myeloperoxidase (MPO) levels, a marker for neutrophil infiltration, ${ }^{28}$ of at least $70 \%$ compared with vehicle-treated and LL-pTREX1-treated mice $(P<0.001$, Figure 4e). Following LL-MT1-MT1 treatment, the mean histological score of the distal colon was improved by at least $25 \%$ compared with the appropriate controls $(P<0.05$, Figure $4 \mathbf{a}, \mathbf{b}, \mathbf{c}$ and $\mathbf{f})$. As expected, LL-mIL10 treatment did not improve the outcome of established enterocolitis in interleukin 10 (IL10) $)^{-/-}$mice (Figure 4d and f).

Summarizing, LL-MT1-MT1 therapy ameliorated the pathology not only in chronic DSS-induced colitis, but also in established chronic colitis in IL10 ${ }^{-/-}$mice.

\section{Comparison between oral LL-MT1-MT1, oral MT1-MT1 and systemic MT1-MT1-AR1 treatment against established chronic enterocolitis in IL10 ${ }^{-1-}$ mice}

Treatment with LL-MT1-MT1 resulted in a histological score of $3.1 \pm 0.4$ in the middle colon, a significant decrease of $40 \%$ compared with empty vector-treated controls ( $P<0.05$; Figure 5).
Oral treatment with MT1-MT1 or systemic treatment with MT1-MT1-AR1 resulted in a histological score of $4.4 \pm 0.7$ and $5.6 \pm 0.5$, respectively, showing no therapeutic benefit compared with the appropriate controls (Figure 5).

\section{Influence of LL-MT1-MT1 therapy on systemic Salmonella} infection in colitic IL10 $10^{-1}$ mice

The spleen of healthy animals contained no Salmonella spp. All other groups received intravenously a sublethal dose of $5 \times 10^{4}$ CFU Salmonella spp. to induce systemic Salmonella infection. After 8 days, LL-MT1-MT1-treated animals had a bacterial load of approximately $1 \times 10^{4} \mathrm{CFU}$ Salmonella spp. per gram spleen, which was equal to the bacterial Salmonella load of vehicle- and empty vector-treated mice (Figure 6). Systemic treatment with anti-TNF immunoglobulin (Ig)G1 antibodies resulted in a fourfold increase of the Salmonella load of the spleen $\left(4.3 \times 10^{4} \mathrm{CFU}\right.$ Salmonella cells per gram spleen, Figure 6) compared with controls and the LL-MT1-MT1-treated group. In contrast to systemic anti-TNF treatment, LL-MT1-MT1 therapy did not influence systemic infection, showing that the effect of $L$. Lactis-secreted anti-mTNF Nanobodies was restricted to the intestine.

\section{DISCUSSION}

This report describes the creation of a new, effective approach for local anti-inflammatory therapy aimed at treating IBD, 

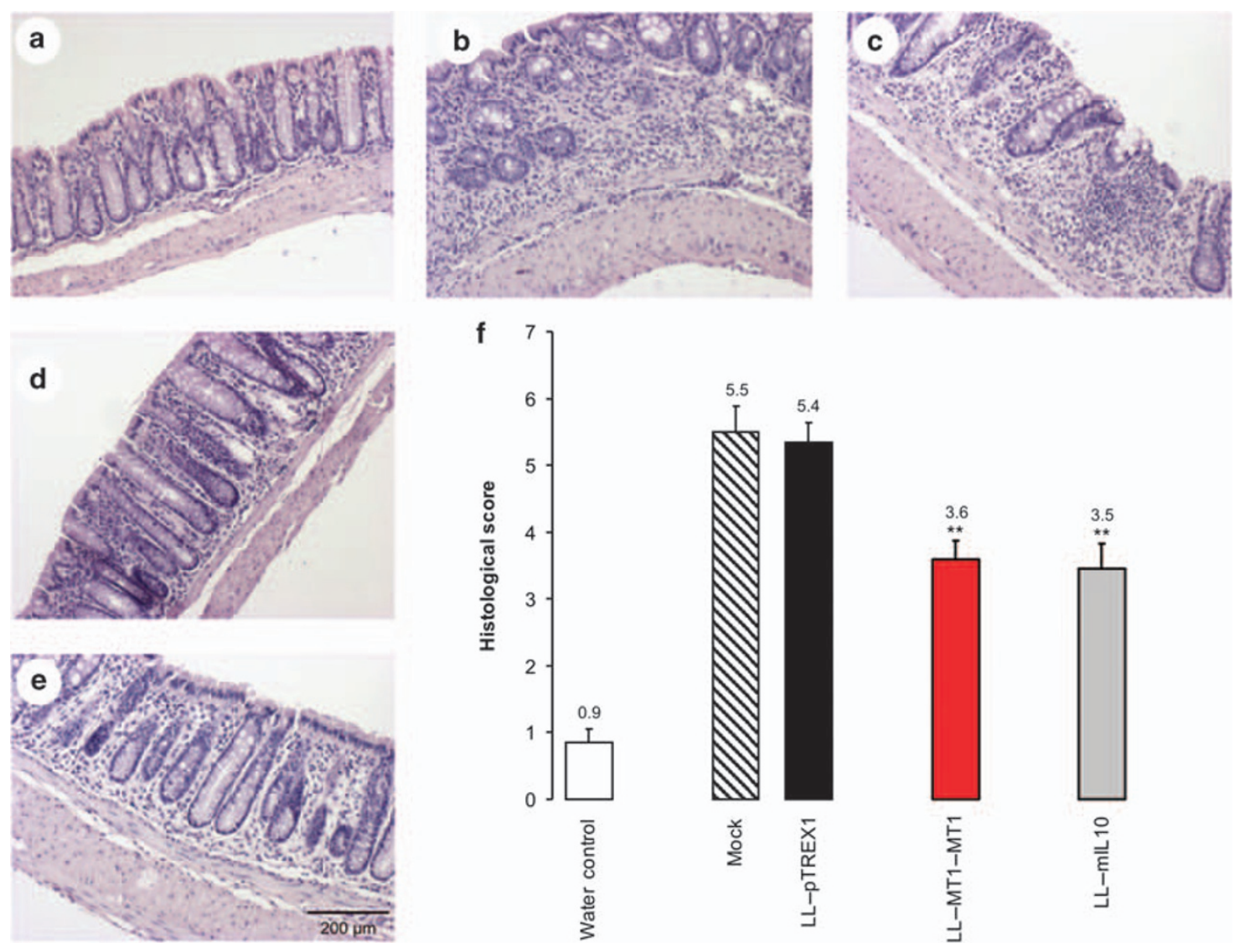

Figure 3 Analysis of morbidity in chronic DSS-induced colitis. (a-e) Representative histology (hematoxylin/eosin staining) of the distal colon from healthy control mice (a) and mice with chronic DSS-induced colitis either mock-treated (b) or treated with LL-pTREX1 (c), LL-MT1-MT1 (d) or LL-mIL10 (e). (f) Statistical evaluation of the histological score of the middle colon. Bars represent the mean \pm s.e.m. Mice with DSS-induced chronic colitis were either Mock-treated or received different $L$. lactis cultures. The Shapiro-Wilk normality test revealed that the groups were not normally distributed. Differences between groups were compared with the Mann-Whitney U-test (Mock-LL-MT1-MT1, P=0.0013; LL-pTREX1—LL-MT1MT1, $P=0.0004$; Mock-LL-mIL10, $P=0.0026$; LL-pTREX1-LL-mIL10, $P=0.0035)$. ${ }^{*}$ Represents a statistically significant difference of $P<0.01$ compared with the vehicle-treated and the empty vector-treated control groups, respectively. DSS, dextran sulfate sodium; L. lactis, Lactococcus lactis.

involving in situ secretion of anti-mTNF Nanobodies by orally administered L. lactis strains.

Intragastric administration of Nanobody-secreting L. lactis resulted in local and active delivery of anti-mTNF Nanobodies at the mucosa of the colon, without measurable levels in systemic circulation. In addition, the Nanobodies were clearly associated with the surface of lamina propria cells, especially in the eroded zones of the mucosa with dominant inflammatory infiltrates.

L. lactis-secreted Nanobodies neutralized both soluble and membrane-bound mTNF in vitro. This could have important therapeutic implications; Nanobodies might bind to tmTNF expressed on the surface of macrophages and T-cells, possibly triggering "reverse signaling" in T-cells and downregulating TNF-driven inflammatory processes in the intestine. ${ }^{29-34}$

Administration of Nanobody-secreting L. lactis strains proved to be highly efficacious in both DSS-induced colitis and established enterocolitis in IL10 ${ }^{-/-}$mice. At least one other study has investigated the possibility of oral delivery of anti-TNF antibodies in mice. Very high doses of orally administered polyclonal avian (yolk) anti-TNF antibodies improved both the acute and chronic phases of induced colitis in rats. ${ }^{34}$ Here, comparable results for orally delivered, purified MT1-MT1 Nanobodies could not be demonstrated. Likely, these were proteolyzed upon digestion. Similar findings were reported by Harmsen et al., ${ }^{35,36}$ who subsequently employed DNA shuffling and stringent selection to establish stable, more resilient Nanobody clones. As this was beyond the scope of our study, comparable engineering and selection was not pursued.

Interestingly, the small quantities of Nanobodies delivered by L. lactis were highly efficacious in reducing signs of (entero)colitis. Similar findings were reported before, showing that topical delivery of trefoil factors by L. lactis was much more efficient than rectal administration of a 1200 -fold higher dose of purified trefoil factors proteins. ${ }^{18}$ Similarly, the L. lactis bacteria engage in intimate contact with the inflamed mucosa, increasing drug availability in close proximity to responsive cells in the epithelium and limiting dilution into the luminal fluid. This contact is facilitated by structural defects in the mucosal lining of the colon, allowing an increase in the number of ActoBiotics in the residual mucus layer and as such passage of the cargo to the underlying lamina propria. Here, only $2 \times 10^{9} \mathrm{CFU} /$ dose was evaluated; one could speculate that increased dosage would boost the therapeutic effect. 

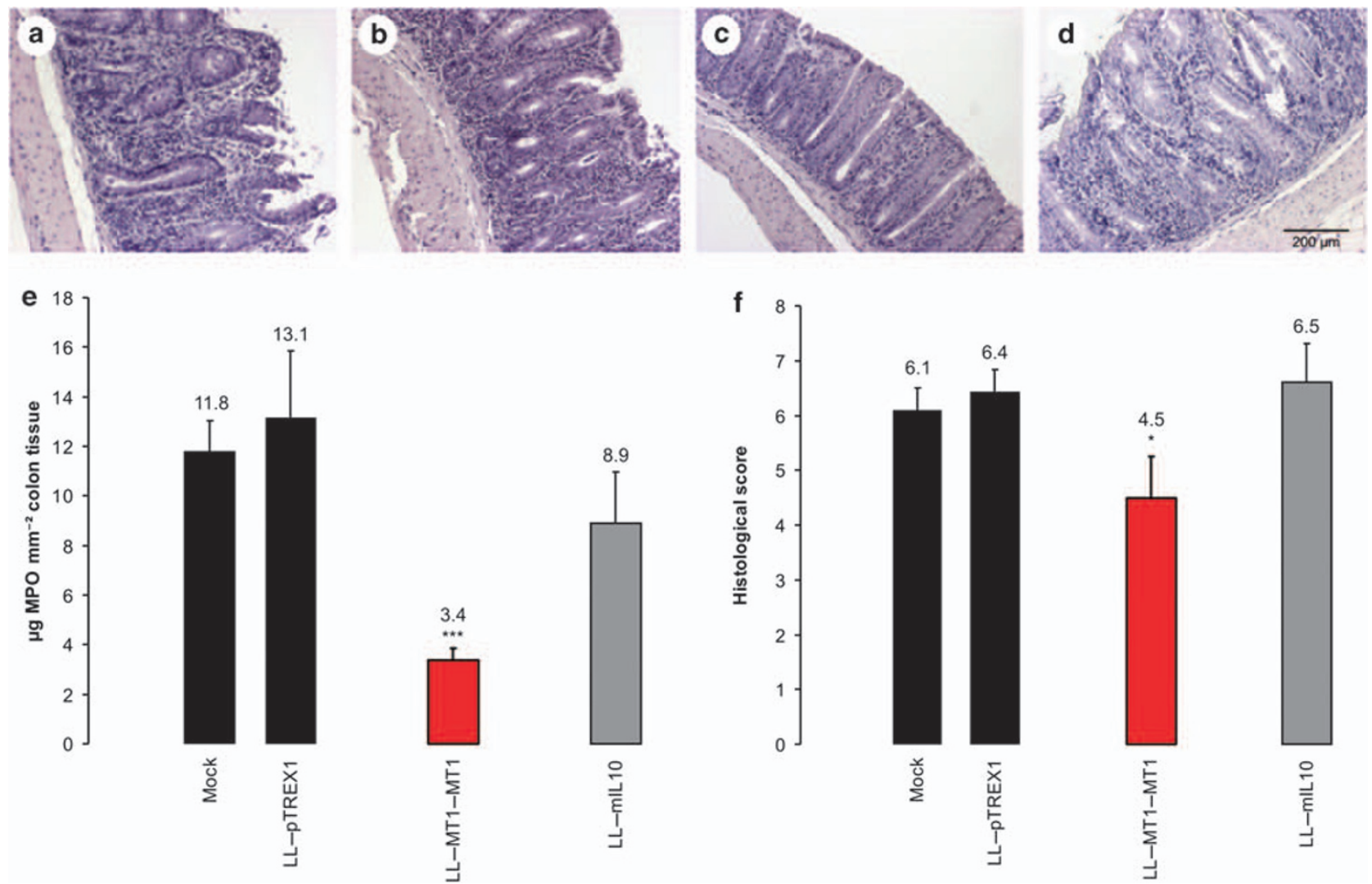

Figure 4 Analysis of morbidity in 20-week-old IL10-/- mice. (a-d) Representative histology (hematoxylin/eosin staining) of the distal colon from IL10 ${ }^{-1-}$ mice, which were mock-treated (a) or treated with LL-pTREX1 (b), LL-MT1-MT1 (c) or LL-mIL10 (d). (e,f) Statistical evaluation of the MPO levels per $\mathrm{mm}^{2}$ colon tissue (e) and histological score of the distal colon (f). Bars represent the mean \pm s.e.m. The Shapiro-Wilk normality test revealed that the groups were not normally distributed. Differences between groups were compared with the Mann-Whitney's U-test (e: Mock-LL-MT1-MT1, $P=0.0002$; LL-pTREX1-LL-MT1-MT1, $P=0.0002 ;$ f: LL-pTREX1, $P=0.0390)$. * And ${ }^{* *}$ represent statistically significant differences of $P<0.05$ and $P<0.001$, respectively, compared with the empty vector-treated control group. IL10, Interleukin 10; MPO, myeloperoxidase.

Several reports also describe systemic administration of anti-TNF antibodies in mice. Intraperitoneal (i.p.) injection of the chimeric anti-mTNF rat/murine IgG2a/K antibody $\mathrm{cV} 1 \mathrm{q}$ in IL10 $0^{-/-}$mice, resulted in a significant improvement of colitis. ${ }^{37,38}$ These findings were not reproduced for systemic MT1-MT1-AR1. However, Nanobody MT1-MT1AR1 was designed for a study in murine collagen-induced arthritis. ${ }^{22}$ Coppieters et al. ${ }^{22}$ engineered MT1-MT1-AR1 to also target albumin by non-covalent association, significantly prolonging serum half-life (from $47 \mathrm{~min}$ to 1.9 days). ${ }^{39}$ A major advantage of this approach was the putative targeting of albumin to inflamed tissues, accumulating in inflamed paws. ${ }^{22}$ However, in IBD, albumin is a typical negative acute phase reactant showing reduced levels during inflammation. ${ }^{40}$ This might have hampered correct targeting and accumulation of systemically applied MT1-MT1-AR1 Nanobodies at the colon.

The experiments reported here have further shown that administration of Nanobody-secreting L. lactis strains did not provoke a systemic immune intervention, in contrast to systemic anti-TNF therapy. Overall, the L. lactis-secreted Nanobodies seem to share the efficacy of traditional anti-TNF therapeutics, while lacking the systemic adverse events. One could also presume that oral intake of L. lactis strains would provide a higher degree of patient comfort compared with the parenteral administration of traditional anti-TNF drugs. Moreover, an oral capsule formulation, specifically developed for ActoBiotics, controls the release of metabolically active L. lactis bacteria, in such a way that it can target inflammation at the lower part of the small intestine as well as in the colon, making it suitable for treatment of Crohn's disease and ulcerative colitis. In addition, the TopAct platform might obviate the need for expensive large scale purification of the anti-TNF therapeutics typically associated with production in eukaryotic cells.

In conclusion, results from this study could lead to a superior and more effective management of colitis in humans without the adverse effects and patient discomfort of traditional antiTNF therapies.

\section{METHODS \\ Bacteria}

L. lactis strain MG1363 was used throughout this study. Bacteria were cultured in GM17E medium, i.e., Difco M17 broth (BD, Franklin Lakes, NJ) supplemented with $0.5 \%$ glucose (Merck KGaA, Darmstadt, Germany) and $5 \mu \mathrm{g} \mathrm{ml}^{-1}$ erythromycin (Sigma, St. Louis, MO). Stock suspensions were stored at $-20^{\circ} \mathrm{C}$ in $50 \%$ glycerol (Merck KGaA) in GM17E. For intragastric inoculations, stock suspensions were diluted 200-fold in fresh GM17E and incubated for $16 \mathrm{~h}$ at $30^{\circ} \mathrm{C}$, reaching a saturation density of $2 \times 10^{9} \mathrm{CFU}$ per ml. Bacteria were harvested by centrifugation and 


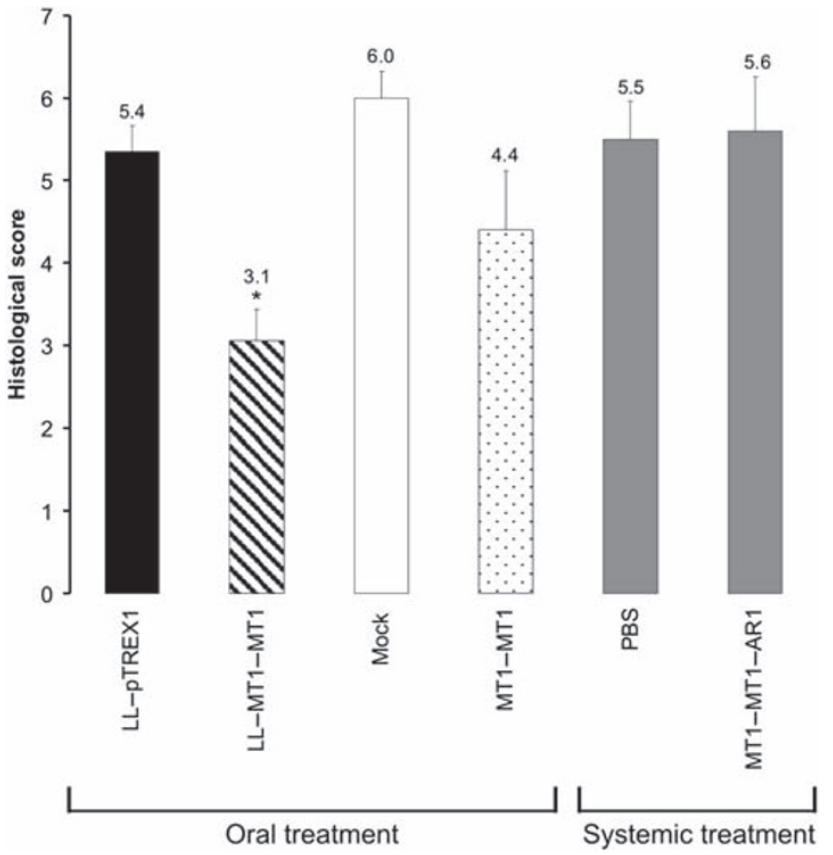

Figure 5 Comparison between oral LL-MT1-MT1, oral MT1-MT1 and systemic MT1-MT1-AR1 treatment against established chronic enterocolitis in IL $10^{-/-}$mice. Statistical evaluation of the histological score of the middle colon of 20 -week-old IL10 ${ }^{-/-}$mice that were treated for 21 days. Bars represent mean \pm s.e.m. The black and hatched bars represent groups that received daily administrations of $2 \times 10^{9} \mathrm{CFU}$ of LL-pTREX1 or LL-MT1-MT1, respectively, through an intragastric catheter. The white and white-spotted bars represent groups that received daily administrations of $100 \mu \mathrm{l} 50 \mathrm{~mm}$ carbonated buffer (mock) or $100 \mu \mathrm{l}$ MT1-MT1 (50 $\left.\mathrm{mg} \mathrm{kg}^{-1}\right)$, respectively, through an intragastric catheter. The gray bars represent groups that were treated every other day by i.p. administration of $100 \mu \mathrm{l}$ PBS or $100 \mu \mathrm{l} \mathrm{MT1-MT1-AR1}\left(25 \mathrm{mg} \mathrm{kg}^{-1}\right)$, respectively. The Shapiro-Wilk's normality test revealed that the groups were normally distributed. Groups were compared with one-way ANOVA followed by HSD multiple comparisons post test. (mock-LL-MT1-MT1, $P=0.012 ;$ LL-pTREX1-LL-MT1-MT1, $P=0.016)$. *Represents a statistically significant difference of $P<0.05$ compared with the mock and vector control LL-pTREX1-treated groups. ANOVA, one-way analysis of variance; CFU, colony forming units; $\mathrm{HSD}$, honestly significant difference; IL10, Interleukin 10; i.p., intraperitoneal; MPO, myeloperoxidase; PBS, phosphate-buffered saline.

concentrated 10-fold in BM9 medium. ${ }^{27}$ Treatment doses consisted of $100 \mu \mathrm{l}$ of this suspension.

\section{Plasmids}

DNA sequences encoding monovalent and bivalent anti-mTNF Nanobodies were provided by Ablynx N.V. (Zwijnaarde, Belgium). ${ }^{22}$ Details of plasmid construction can be obtained from the authors. MG1363 strains transformed with plasmids carrying the MT1 or MT1MT1 Nanobody coding sequence extended with a HisG and Myc-tag, were designated LL-MT1 and LL-MT1-MT1, respectively. LL-pTREX1, an L. lactis MG1363 strain containing the empty vector pTREX1, served as control. LL-mIL10 was an L. lactis MG1363 strain containing plasmid pT1mIL10 and constitutively secreted mIL10. ${ }^{17}$

\section{MT1 and MT1-MT1 quantification}

Myc-tagged MT1 and MT1-MT1 Nanobodies, secreted in vitro, were quantified by direct adsorption of crude L. lactis supernatants to a NuncImmuno Maxisorp F96 microtiterplate (Nunc, New York, NY) and subsequent detection with a specific mouse monoclonal antibody against the Myc epitope (Sigma).
For quantification of MT1 and MT1-MT1 Nanobodies secreted in vivo in colon tissue, 10 serial inoculations of either $100 \mu \mathrm{l}$ phosphate-buffered saline (PBS) or $100 \mu \mathrm{l}$ suspensions containing $2 \times 10^{9} \mathrm{CFU}$ LL-pTREX1, LL-MT1 or LL-MT1-MT1, respectively were administered to female $\mathrm{BALB} / \mathrm{c}$ mice $(n=2)$ with DSS-induced colitis at intervals of $30 \mathrm{~min}$. One hour after the last inoculation, blood was sampled and the colon excised. A tissue sample was taken form the distal part of the colon for immunohistochemistry, the remainder was homogenized in PBS containing $1 \%$ bovine serum albumin (BSA, Sigma) and complete protease inhibitor (Roche Diagnostics GmbH, Mannheim, Germany) and subsequently sonicated. MT1-MT1 Nanobodies were measured in the colon homogenate using the Nanobody quantification protocol.

\section{Nanobody quantification protocol}

A Nunc-Immuno Maxisorp F96 microtiterplate was coated with $1 \mu \mathrm{g} \mathrm{ml}^{-1}$ mTNF (PSF-VIB, Zwijnaarde, Belgium) overnight at $4^{\circ} \mathrm{C}$. The plate was washed and blocked. A standard curve was made with MT1 or MT1-MT1 Nanobodies at a start concentration of $1 \mu \mathrm{g} \mathrm{ml}^{-1}$. Dilutions were made in PBS containing 1\% mouse plasma. The Nanobodies were allowed to bind for $2 \mathrm{~h}$ at room temperature. The plate was washed five times and rabbit polyclonal anti-Nanobody antibody K208 was applied at 3000-fold dilution for $1 \mathrm{~h}$ at room temperature. After washing, binding was detected with goat polyclonal anti-rabbit horseradish peroxidase (HRP; DAKO, Glostrup, Denmark) at a 3000-fold dilution for $1 \mathrm{~h}$ at room temperature and stained with tetramethylbenzidine substrate (BD). Reaction was stopped by adding $1 \mathrm{M} \mathrm{H}_{2} \mathrm{SO}_{4}$ (Merck KGaA). The optical density at $450 \mathrm{~nm}$ (OD450 nm) was measured, with OD595 nm as reference wavelength.

\section{Soluble TNF and tmTNF neutralization assay}

The inhibitory effect of MT1 and MT1-MT1 Nanobodies on soluble mTNF $\left(20 \mathrm{IU} \mathrm{ml}^{-1}\right)$ was measured in an $18 \mathrm{~h}$ cytotoxicity assay using murine fibroblast WEHI 164 clone 13-cells in the presence of $1 \mu \mathrm{g} \mathrm{ml}^{-1}$ actinomycin D (Invitrogen, Carlsbad, CA), as described. ${ }^{41}$ E. coli-produced, purified MT1 and MT1-MT1 were used as reference.

The inhibitory effect of MT1 and MT1-MT1 on the cytotoxic effect of tmTNF was determined by a co-culture between the transfected, uncleavable tmTNF-expressing L929 effector cells and TNF-responsive target cells (WEHI 164 clone 13), as described. ${ }^{42}$

\section{Animals}

11-week-old female BALB/c mice were obtained form Charles River Laboratories Italy S.r. 1. (Calco, Italy). IL10 knockout mice (129Sv/Ev IL $10^{-/-}$) were bred under specific pathogen-free (SPF) conditions. The IL $10^{-/-}$mice were used at 20 weeks of age, at which time enterocolitis had fully developed. ${ }^{43}$ All mice were housed under SPF conditions and fed standard laboratory feed and tap water ad libitum. All studies were approved by the Ethics Committee of the Department for Molecular Biomedical Research, Ghent University (File No. 04/02 and 04/03).

\section{Induction of chronic colitis by DSS}

Chronic colitis was induced in mice weighing approximately $21 \mathrm{~g}$ by four cycles of administration of 5\% (weight/volume) DSS (40 kDa, Applichem, Darmstadt, Germany) in the drinking water, alternating with 10-day periods of recovery. ${ }^{44}$

\section{Therapeutic effect of LL-MT1-MT1 against DSS-induced chronic colitis}

Treatment was arbitrarily initiated at day 21 after the last DSS administration and lasted for 21 days. Healthy mice receiving normal drinking water were used as healthy controls. Four groups of mice received intragastric inoculations with either vehicle alone or vehicle containing $2 \times 10^{9} \mathrm{CFU}$ of vector control LL-pTREX1, bivalent Nanobody-secreting LL-MT1-MT1 or mIL10-secreting LL-mIL10, respectively ( $n=10$ for all groups). $2 \times 10^{9} \mathrm{CFU}$ represents a technically maximal reliable dose for freshly cultured strains. Seven days after the treatment period, mice were killed and analyzed.

Therapeutic effect of LL-MT1-MT1 against established chronic enterocolitis in $\mathrm{IL}_{10}{ }^{-/-}$mice. The effect of LL-MT1-MT1 treatment 


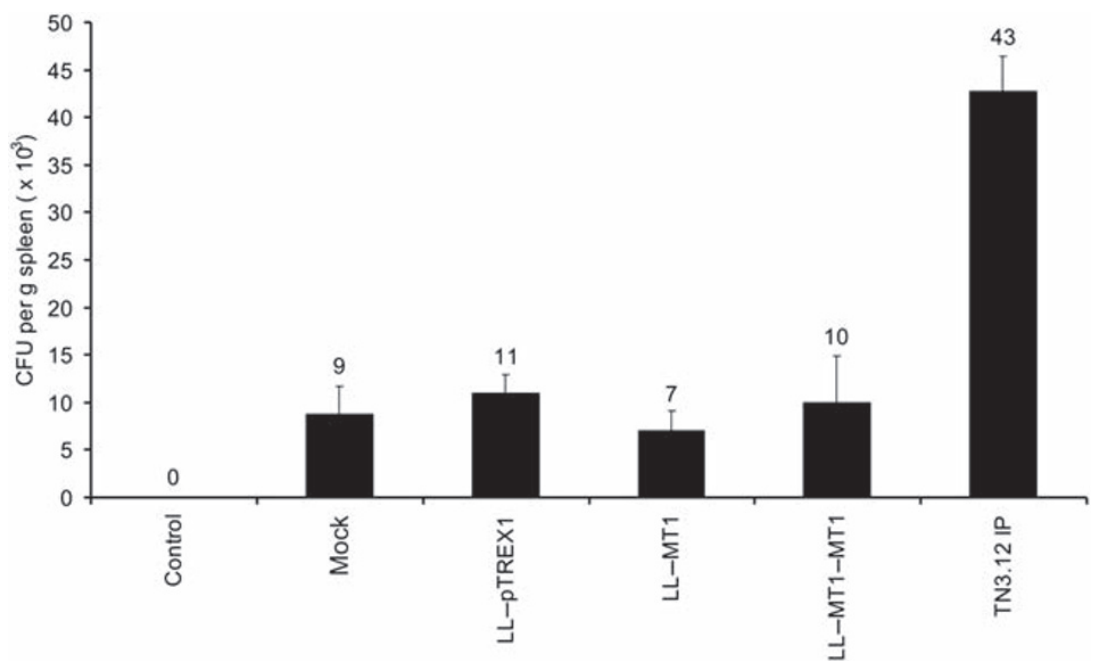

Figure 6 Influence of LL-MT1-MT1 therapy on systemic Salmonella infection in colitic IL10 $10^{-/}$mice. All groups received Salmonella cells, except control mice. TN3-19.12 hamster IgG1 anti-TNF monoclonal antibody given i.p. once every 3 days was used as positive control to mimic systemic anti-TNF treatment. Ig, immunoglobulin; i.p., intraperitoneal; TNF, tumor necrosis factor.

on spontaneously developing chronic enterocolitis in IL10 $10^{-/-}$mice was evaluated. Twenty-week-old mice were treated for 14 days by daily intragastric inoculation with either vehicle or $2 \times 10^{9} \mathrm{CFU}$ of LL-pTREX1, LL-MT1-MT1 or LL-mIL10 ( $n=8$ for all groups). All mice were killed the day after the final administration.

\section{Comparison between oral LL-MT1-MT1, oral MT1-MT1 and systemic MT1-MT1-AR1 treatment against established chronic enterocolitis in IL10-1- mice.}

To compare the therapeutic efficacy of oral LL-MT1-MT1 administration with oral MT1-MT1 and systemic MT1-MT1-AR1 administration (systemically applied MT1-MT1 fused to anti-serum albumin has a more acceptable half-life in mice than MT1-MT1), ${ }^{22} 20$-week-old IL10 ${ }^{-1-}$ mice were treated for 21 days. Two groups of mice received daily intragastric inoculations of $2 \times 10^{9} \mathrm{CFU}$ vector control LL-pTREX1 or LL-MT1-MT1, respectively ( $n=10$ for both). Two other groups of mice received daily intragastric inoculations of $100 \mu \mathrm{l} 50 \mathrm{~mm}$ carbonate buffer (mock; $n=9$ ) or $100 \mu \mathrm{l} \mathrm{MT1-MT1}\left(50 \mathrm{mg} \mathrm{kg}^{-1} ; n=10\right)$, respectively. Finally, two groups of mice were treated every other day by i.p. administration of $100 \mu \mathrm{l}$ PBS $(n=8)$ or $100 \mu \mathrm{l} \mathrm{MT1-MT1-AR1}\left(25 \mathrm{mg} \mathrm{kg}^{-1} ; n=8\right)$, respectively. All mice were killed the day after the last treatment.

\section{MPO assay}

MPO activity in the middle colon was measured as described. ${ }^{28}$ Pure human MPO was used as standard (Calbiochem-Merck KGaA, Darmstadt, Germany). Data are expressed as $\mu \mathrm{g} \mathrm{MPO} \mathrm{mm}^{-2}$ colon tissue.

\section{Histological analysis}

For histological analysis, the colon was removed, cleaned, and opened longitudinally. A segment of $1 \mathrm{~cm}$ was taken from the distal part, embedded in paraffin and sectioned longitudinally. Three sections of $4 \mu \mathrm{m}$ were cut at $200 \mu \mathrm{m}$ intervals and stained with hematoxylin/eosin. Colon sections were numbered randomly and interpreted semi-quantitatively in a blinded manner. The histological score is the sum of the epithelial damage and lymphoid infiltration, each ranging from 0 to 4 as described. ${ }^{45}$

\section{Immunohistochemistry}

Paraffin-embedded colon sections were incubated with polyclonal rabbit anti-Nanobody antibody K208 (1/2,000). A secondary biotinylated swine anti-rabbit antibody $(1 / 400 ; \mathrm{DAKO})$ was revealed with HRP-conjugated streptavidin (streptAB complex/HRP; DAKO) and subsequent reaction with 3-amino-9-ethylcarbazole (AEC) substrate-chromogen (DAKO). The sections were stained afterwards with hematoxylin.

\section{Systemic Salmonella infection in colitic IL10 ${ }^{-1-}$ mice}

A sublethal dose of $5 \times 10^{4} \mathrm{CFU}$ Salmonella enteritica subsp. enterica serovar Thyphimurium (BCCM/LMG strain 3264, Ghent, Belgium) was injected intravenously in the caudal vein of $\mathrm{IL} 10^{-/-}$mice to induce systemic Salmonella infection. The infected IL $10^{-/-}$mice were treated for 7 days and received $100 \mu \mathrm{l}$ daily of either vehicle alone or vehicle containing $2 \times 10^{9}$ LL-pTREX1 or LL-MT1-MT1, respectively ( $n=3$ in each group) through an intragastric catheter. As positive control, $100 \mu \mathrm{g}$ TN3-19.12 hamster IgG1 anti-TNF monoclonal antibody (eBioscience Inc., San Diego, $\mathrm{CA})^{46}$ was given i.p. once every 3 days (on day 1,4 , and $7 ; n=3$ ) to mimic systemic anti-TNF treatment. ${ }^{46}$ All mice were euthanized on day 8 . Bacterial Salmonella count of the spleen was used as a read-out for systemic infection of the reticuloendothelial system. Spleen was isolated in an aseptic manner, homogenized in PBS, and plated out on Difco Salmonella-Shigella (SS) plates (BD), after which colonies were counted.

\section{Statistical analysis}

Normality was evaluated with the Shapiro-Wilk's test. Normally distributed groups were compared with one-way analysis of variance followed by honestly significant difference multiple comparisons post test. Data that were not normally distributed were analyzed with the Mann-Whitney $U$-test (two-tailed).

\section{ACKNOWLEDGMENTS}

We thank I. Bruggeman, H. Devlies, M. Van Den Hemel, and K. Van Laer for technical assistance, J. Wells for the vector PTREX1 and P. Hermans (Unilever Research, Vlaardingen, The Netherlands) for providing rabbit anti-Nanobody serum. Writing assistance was provided by Tim De Smedt, Medical and Scientific Writer at ActoGeniX N.V. K. Vandenbroucke held a postdoctoral grant from the Broad Medical Research Project. This research was funded by Broad Medical Research Project and VIB. The trademarks TopAct and ActoBiotics are used with the kind permission of ActoGeniX N.V. Nanobody and Nanobodies are used with the kind permission of Ablynx N.V.

\section{DISCLOSURE}

The authors affiliated with ActoGeniX N.V. and Ablynx N.V. have financial interests in ActoGeniX N.V. and Ablynx N.V.,respectively, including employment and stock options.

() 2010 Society for Mucosal Immunology 


\section{REFERENCES}

1. Bouma, G. \& Strober, W. The immunological and genetic basis of inflammatory bowel disease. Nat. Rev. Immunol. 3, 521-533 (2003).

2. Papadakis, K.A. \& Targan, S.R. Tumor necrosis factor: biology and therapeutic inhibitors. Gastroenterology 119, 1148-1157 (2000).

3. Vassalli, P. The pathophysiology of tumor necrosis factors. Annu. Rev. Immunol. 10, 411-452 (1992)

4. van Dullemen, H.M. et al. Treatment of Crohn's disease with anti-tumor necrosis factor chimeric monoclonal antibody (cA2). Gastroenterology 109, 129-135 (1995).

5. Hanauer, S.B. et al. Maintenance infliximab for Crohn's disease: the ACCENT I randomised trial. Lancet 359, 1541-1549 (2002).

6. Rutgeerts, P., Van Assche, G. \& Vermeire, S. Optimizing anti-TNF treatment in inflammatory bowel disease. Gastroenterology 126, 15931610 (2004).

7. Rutgeerts, P. et al. Infliximab for induction and maintenance therapy for ulcerative colitis. N. Engl. J. Med. 353, 2462-2476 (2005).

8. Riegert-Johnson, D.L. et al. Delayed hypersensitivity reaction and acute respiratory distress syndrome following infliximab infusion. Inflamm. Bowel Dis. 8, 186-191 (2002).

9. Ali, Y. \& Shah, S. Infliximab-induced systemic lupus erythematosus. Ann. Intern. Med. 137, 625-626 (2002).

10. Chung, E.S., Packer, M., Lo, K.H., Fasanmade, A.A. \& Willerson, J.T. Randomized, double-blind, placebo-controlled, pilot trial of infliximab, a chimeric monoclonal antibody to tumor necrosis factor-alpha, in patients with moderate-to-severe heart failure: results of the anti-TNF Therapy Against Congestive Heart Failure (ATTACH) trial. Circulation 107, 31333140 (2003).

11. Colombel, J.F. et al. The safety profile of infliximab in patients with Crohn's disease: the Mayo clinic experience in 500 patients. Gastroenterology 126, 19-31 (2004).

12. Andus, T., Stange, E.F., Hoffler, D. \& Keller-Stanislawski, B. [Suspected cases of severe side effects after infliximab (Remicade) in Germany]. Med. Klin. (Munich) 98, 429-436 (2003).

13. Keane, J. et al. Tuberculosis associated with infliximab, a tumor necrosis factor alpha-neutralizing agent. N. Engl. J. Med. 345, 1098-1104 (2001).

14. Rutgeerts, P. et al. Efficacy and safety of retreatment with anti-tumor necrosis factor antibody (infliximab) to maintain remission in Crohn's disease. Gastroenterology 117, 761-769 (1999).

15. Farrell, R.J. et al. Intravenous hydrocortisone premedication reduces antibodies to infliximab in Crohn's disease: a randomized controlled trial. Gastroenterology 124, 917-924 (2003).

16. Baert, F. et al. Influence of immunogenicity on the long-term efficacy of infliximab in Crohn's disease. N. Engl. J. Med. 348, 601-608 (2003).

17. Steidler, L. et al. Treatment of murine colitis by Lactococcus lactis secreting interleukin-10. Science 289, 1352-1355 (2000).

18. Vandenbroucke, K. et al. Active delivery of trefoil factors by genetically modified Lactococcus lactis prevents and heals acute colitis in mice. Gastroenterology 127, 502-513 (2004).

19. Huibregtse, I.L. et al. Induction of ovalbumin-specific tolerance by oral administration of Lactococcus lactis secreting ovalbumin. Gastroenterology 133, 517-528 (2007).

20. Steidler, L. et al. Biological containment of genetically modified Lactococcus lactis for intestinal delivery of human interleukin 10. Nat. Biotechnol. 21, 785-789 (2003).

21. Braat, $H$. et al. A phase I trial with transgenic bacteria expressing Interleukin10 in Crohn's disease. Clin. Gastroenterol. Hepatol. 4, 754-759 (2006).

22. Coppieters, K. et al. Formatted anti-Tumor Necrosis Factor alpha VHH proteins derived from camelids show superior potency and targeting to inflamed joints in a murine model of collagen-induced arthritis. Arthritis Rheum. 54, 1856-1866 (2006).

23. Lauwereys, M. et al. Potent enzyme inhibitors derived from dromedary heavy-chain antibodies. EMBO J. 17, 3512-3520 (1998).

24. Arbabi Ghahroudi, M., Desmyter, A., Wyns, L., Hamers, R. \& Muyldermans, $S$. Selection and identification of single domain antibody fragments from camel heavy-chain antibodies. FEBS Lett. 414, 521-526 (1997).
25. Frenken, L.G. et al. Isolation of antigen specific llama VHH antibody fragments and their high level secretion by Saccharomyces cerevisiae. J. Biotechnol. 78, 11-21 (2000).

26. Els Conrath, K., Lauwereys, M., Wyns, L. \& Muyldermans, S. Camel single-domain antibodies as modular building units in bispecific and bivalent antibody constructs. J. Biol. Chem. 276, 7346-7350 (2001)

27. Schotte, L., Steidler, L., Vandekerckhove, J. \& Remaut, E. Secretion of biologically active murine interleukin-10 by Lactococcus lactis. Enzyme Microb. Technol. 27, 761-765 (2000).

28. Bradley, P.P., Priebat, D.A., Christensen, R.D. \& Rothstein, G. Measurement of cutaneous inflammation: estimation of neutrophil content with an enzyme marker. J. Invest. Dermatol. 78, 206-209 (1982).

29. Eissner, G. et al. Reverse signaling through transmembrane TNF confers resistance to lipopolysaccharide in human monocytes and macrophages. J. Immunol. 164, 6193-6198 (2000).

30. Eissner, G., Kolch, W. \& Scheurich, P. Ligands working as receptors: reverse signaling by members of the TNF superfamily enhance the plasticity of the immune system. Cytokine Growth Factor Rev. 15, 353366 (2004)

31. Mitoma, H. et al. Infliximab induces potent anti-inflammatory responses by outside-to-inside signals through transmembrane TNF-alpha. Gastroenterology 128, 376-392 (2005).

32. Knight, D.M. et al. Construction and initial characterization of a mousehuman chimeric anti-TNF antibody. Mol. Immunol. 30, 1443-1453 (1993).

33. Mitoma, H., Horiuchi, T. \& Tsukamoto, H. Binding activities of infliximab and etanercept to transmembrane tumor necrosis factor-alpha. Gastroenterology 126, 934-935 author reply 935-936 (2004).

34. Worledge, K.L., Godiska, R., Barrett, T.A. \& Kink, J.A. Oral administration of avian tumor necrosis factor antibodies effectively treats experimental colitis in rats. Dig. Dis. Sci. 45, 2298-2305 (2000).

35. Harmsen, M.M. et al. Escherichia coli F4 fimbriae specific llama singledomain antibody fragments effectively inhibit bacterial adhesion in vitro but poorly protect against diarrhoea. Vet. Microbiol. 111, 89-98 (2005).

36. Harmsen, M.M., Van Solt, C.B., van Zijderveld-van Bemmel, A.M., Niewold, T.A. \& van Zijderveld, F.G. Selection and optimization of proteolytically stable llama single-domain antibody fragments for oral immunotherapy. Appl. Microbiol. Biotechnol. 72, 544-551 (2006).

37. Gratz, R. et al. Murine monoclonal anti-tNF antibody administration has a beneficial effect on inflammatory bowel disease that develops in IL-10 knockout mice. Dig. Dis. Sci. 47, 1723-1727 (2002).

38. Scheinin, T., Butler, D.M., Salway, F., Scallon, B. \& Feldmann, M. Validation of the interleukin-10 knockout mouse model of colitis: antitumour necrosis factor-antibodies suppress the progression of colitis. Clin. Exp. Immunol. 133, 38-43 (2003).

39. Dennis, M.S. et al. Albumin binding as a general strategy for improving the pharmacokinetics of proteins. J. Biol. Che 277, 35035-35043 (2002).

40. Vermeire, S., Van Assche, G. \& Rutgeerts, P. Laboratory markers in IBD: useful, magic, or unnecessary toys? Gut 55, 426-431 (2006).

41. Espevik, T. \& Nissen-Meyer, J. A highly sensitive cell line, WEHI 164 clone 13 , for measuring cytotoxic factor/tumor necrosis factor from human monocytes. J. Immunol. Methods 95, 99-105 (1986).

42. Decoster, E., Vanhaesebroeck, B., Vandenabeele, P., Grooten, J. \& Fiers, W. Generation and biological characterization of membrane-bound, uncleavable murine tumor necrosis factor. J. Biol. Chem. 270, $18473-$ 18478 (1995).

43. Kuhn, R., Lohler, J., Rennick, D., Rajewsky, K. \& Muller, W. Interleukin-10deficient mice develop chronic enterocolitis. Cell 75, 263-274 (1993).

44. Okayasu, l. et al. A novel method in the induction of reliable experimental acute and chronic ulcerative colitis in mice. Gastroenterology 98, 694702 (1990).

45. Kojouharoff, G. et al. Neutralization of tumour necrosis factor (TNF) but not of IL-1 reduces inflammation in chronic dextran sulphate sodium-induced colitis in mice. Clin. Exp. Immunol. 107, 353-358 (1997).

46. Sheehan, K.C., Ruddle, N.H. \& Schreiber, R.D. Generation and characterization of hamster monoclonal antibodies that neutralize murine tumor necrosis factors. J. Immunol. 142, 3884-3893 (1989). 